\title{
Non-equilibrium of populations with a mixed breeding strategy
}

\author{
Poroshina A.A. \\ Limnological Institute, SB RAS, Irkutsk, Russia \\ a.poroshina@lin.irk.ru
}

Key words: microsatellite markers, object-oriented modeling, asexual reproduction, parthenogenesis

Motivation and Aim: The pattern of genetic diversity in populations with sexual reproduction is shaped by the recombination and independent segregation of genetic material in several generations. This leads to the formation of new combinations of alleles. Some of the new alleles that have arisen as a result of spontaneous mutations are fixed or eliminated from the population due to gene drift and/or natural selection. In vegetatively propagating populations, the process of recombination and segregation of alleles is absent. For this reason, during vegetative reproduction, the genotype is transferred from one individual to another completely. In this case, genetic diversity is formed only due to the mutational process $[1,2]$ and competition/drift of clones.

Methods and Algorithms: In our work, we tried to investigate how the transition from sexual reproduction to vegetative reproduction will affect the population of diploid organisms with a neutral character of molecular evolution. At the same time, special attention was paid to the specificity of microsatellite markers. We described the general picture of violation of the Hardy-Weinberg principle using the methods of objectoriented simulation modeling.

Results: According to the tables of the stored intermediate results for the populations, the indicators of the expected heterozygosity of $\mathrm{He}$ were calculated. The observed heterozygosity - Ho was estimated directly as the proportion of heterozygous individuals in the sample. The population parameter $\theta$ and average $\mathrm{He} / \mathrm{Ho}$ values were also estimated.

Conclusion: From all of the above, we can conclude that the analysis should take into account the effective population size, the lower the value of the mutation probability, the greater the difference between the observed and expected heterozygosity. The differences are more pronounced in asexual sexual reproduction. The average values of the $\theta$-parameter in apomixis populations are much higher than the average values in sexually reproducing populations. The more states at a locus, the stronger the difference between the scatter of expected and observed values. Asexual reproduction helps preserve genetic diversity in a population.

\section{References}

1. Page R.D., Holmes E.C. Molecular Evolution: A Phylogenetic Approach. John Wiley Sons, 2009;1(1):120-125.

2. Wang I.J., Bradburd G.S. Isolation by environment. Mol. Ecol. 2014;23(23):5649-5662. 